\title{
Pseudo 3D Imaging of Dielectric and Magnetic Anomalies from GPR Data
}

\author{
Raffaele Persico, ${ }^{1}$ Sergio Negri, ${ }^{2}$ Francesco Soldovieri, ${ }^{3}$ and Elena Pettinelli ${ }^{4}$ \\ ${ }^{1}$ Institute for Archaeological and Monumental Heritage (IBAM), CNR, 73100 Lecce, Italy \\ ${ }^{2}$ Department of Biological and Environmental Sciences and Technologies, University of Salento, 73100 Lecce, Italy \\ ${ }^{3}$ Institute for Electromagnetic Sensing of the Environment (IREA), CNR, 80124 Naples, Italy \\ ${ }^{4}$ Department of Physics, Roma Tre University, 00146 Roma, Italy
}

Correspondence should be addressed to Francesco Soldovieri, soldovieri.f@irea.cnr.it

Received 12 March 2012; Accepted 25 June 2012

Academic Editor: Nicola Masini

Copyright ( $) 2012$ Raffaele Persico et al. This is an open access article distributed under the Creative Commons Attribution License, which permits unrestricted use, distribution, and reproduction in any medium, provided the original work is properly cited.

This paper deals with the reconstruction of buried targets exhibiting both dielectric and magnetic characteristics, starting from GPR data collected at the interface air/soil. The problem is tackled under the Born approximation. In particular, two-dimensional migration and linear inversion results will be compared versus experimental data and three-dimensional representations of the reconstructions achieved from both methods will be shown.

\section{Introduction}

GPR data processing has been historically focused on dielectric anomalies $[1,2]$, whereas the magnetic properties of the targets and of the embedding medium are customarily neglected. Recently, it has been outlined that the anomalies and/or the background scenario can exhibit magnetic properties, even in the radio-frequency and microwave range [3-9].

For this reason, in previous papers $[10,11]$ the problem of the estimation of dielectric and magnetic anomalies has been theoretically studied. It has been worked out that, at least within a 2D linear inversion model, based on the Born approximation and specifically referred to common offset GPR prospecting, the magnetic nature of the targets is not retrievable in absence of a priori information. Therefore, if one is interested in discriminating whether the targets have or not magnetic properties, either a more complicated model (where nonlinear effects and/or polarization effects are accounted for) or further measurements (i.e., not only GPR data) are useful. The theoretical demonstration passes through a long series of mathematical passages and will not repeated here. In [12], experimental data with magnetic targets have been gathered and processed for the first time. In that occasion, however, only four B-scans on the targets were gathered.

In this paper, we present reconstruction results by considering a measurement survey on a more complete grid in order to achieve pseudo $3 \mathrm{D}$ reconstructions with a specific focus on the depth slices. In particular, we have made use of a tank filled with sand, which was relatively wet at the time of the first measurements (exploited in [12]) but was quite drier when we have gathered the data shown here.

The paper is organised as follows. In Section 2 we present the generalized two-dimensional scalar scattering equations, relative to dielectric and magnetic objects embedded in a dielectric and magnetic (possibly lossy) soil. In Section 3, we briefly resume the theoretical discussion about the expected effects of the possible magnetic properties of the buried object on the reconstruction. In Section 4 the experimental case is shown. Conclusions follow.

\section{Formulation of the Problem}

The reference scenario is composed by two half spaces, with the interface air/soil at $z=0$, according to Figure 1 . The 


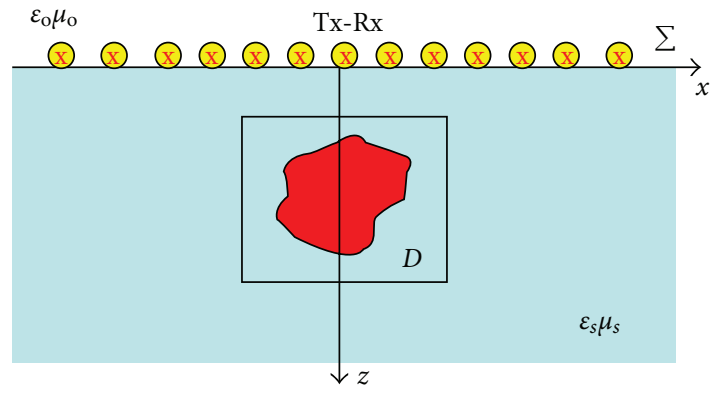

Figure 1: Geometry of the problem.

upper medium is built of air (assimilated to vacuum space), and the lower one shows an equivalent dielectric permittivity $\varepsilon_{s}$ and magnetic permeability $\mu_{s}$, both possibly complex to account for losses.

As source, we consider a filamentary electric current $I_{o}$ invariant along the $y$-axis. The measurement configuration is a common offset B-scan with a fixed offset $\Delta$ between source and receiver. The source moves along the air-soil interface within the observation domain $\Sigma=\left[-x_{M}, x_{M}\right]$. The targets are enclosed in the investigation domain $D=[-a, a] \times$ $\left[z_{\min }, z_{\min }+2 b\right]$, where $a$ is the semiextent along the $x$-axis; $z_{\min }$ and $b$ are the minimum depth and the extent of the investigation domain, along the $z$-axis, respectively.

The problem can be recast in terms of the dielectric and magnetic contrast functions, respectively, defined as

$$
\begin{aligned}
\chi_{e}(x, z) & =\frac{\varepsilon(x, z)-\varepsilon_{s}}{\varepsilon_{s}}, \\
\chi_{m}(x, z) & =\frac{\mu(x, z)-\mu_{s}}{\mu_{s}},
\end{aligned}
$$

where $\varepsilon(x, z)$ is the equivalent dielectric permittivity and $\mu(x, z)$ is the equivalent magnetic permeability within the investigation domain $D$, respectively. The contrast functions in (1) account for the targets as "anomalies" with respect to the background scenario.

The scattering equations worked out are expressed as follows:

$$
\begin{aligned}
& E=E_{\mathrm{inc}}+k_{s}^{2} \iint_{D}\left[\frac{1}{k_{s}^{2}} \nabla^{\prime} G_{i} \cdot \nabla^{\prime} E \frac{\chi_{m}}{1+\chi_{m}}+G_{i} E \chi_{e}\right] d x^{\prime} d z^{\prime} \\
& E_{s}=k_{s}^{2} \iint_{D}\left[\frac{1}{k_{s}^{2}} \nabla^{\prime} G_{e} \cdot \nabla^{\prime} E \frac{\chi_{m}}{1+\chi_{m}}+G_{e} E \chi_{e}\right] d x^{\prime} d z^{\prime}
\end{aligned}
$$

Equations (2) and (3) extend the classical two-dimensional scattering equations $[1,2]$ because of the magnetic terms. In particular, (2) accounts for the electromagnetic field inside the investigation domain, whereas (3) accounts for the scattered field, that is, the datum of our problem. Accordingly, in (2) and (3) $G_{i}\left(x, z, x^{\prime}, z^{\prime}\right)$ and $G_{e}\left(x, z, x^{\prime}, z^{\prime}\right)$ are the internal and external Green's function. The gradient symbols are primed because they refer to $x^{\prime}$ and $z^{\prime}$ meant with respect to the primed couple of variables (the spatial variables defined in the investigation domain D). Moreover, $E$ and $E_{\text {inc }}$ are the ( $y$-directed) internal and incident electric fields, whereas $E_{s}$ is the ( $y$-directed) scattered field. The dot symbol in (2) and (3) stands for "scalar products," and, finally, $k_{o}$ and $k_{s}$ are the wavenumbers in the air and in the soil.

\section{Diffraction Tomography Relationships}

Diffraction tomography (DT) provides well-known [13] approximate linear algebraic relationships between the spectra of the contrast and the one of the scattered field. We resume here the extension of the DT relationships to the more general case with both dielectric and magnetic anomalies; this analysis is discussed in detail in $[11,12]$.

The analysis is based on the following assumptions:

$$
\begin{gathered}
E(x, z) \approx E_{\mathrm{inc}}, \\
\nabla E(x, z) \approx \nabla E_{\mathrm{inc} .}
\end{gathered}
$$

Equations (4) stem from the Born approximation in presence of both dielectric and magnetic anomalies. Under the above assumptions, we can rewrite (3) as

$$
E_{s} \approx k_{s}^{2} \iint_{D}\left[\frac{1}{k_{s}^{2}} \nabla^{\prime} G_{e} \nabla^{\prime} E_{\mathrm{inc}} \chi_{m 1}+G_{e} E_{\mathrm{inc}} \chi_{e}\right] d x^{\prime} d z^{\prime},
$$

where

$$
\chi_{m 1} \equiv \frac{\chi_{m}}{1+\chi_{m}}
$$

is a "derived" magnetic contrast function.

Substituting in (5) the expressions of the external Green function and of the incident field due to a filamentary current (available, i.e., in [11]), the generalised DT relationship for dielectric and magnetic anomalies is then worked out as

$$
\widehat{E}_{s}(p)=f(p) \hat{\hat{\chi}}_{e 1}(\eta(p), \varsigma(p))+f(p) \widehat{\hat{\chi}}_{m 11}(\eta(p), \varsigma(p)),
$$

where $\hat{E}_{s}(p)$ is the Fourier transform of the scattered field with respect to the abscissa $x_{s}$ of the source point (variable along the observation line $\Sigma), \hat{\hat{\chi}}_{e 1}(\eta(p), \varsigma(p))$ is the double Fourier transform of the auxiliary function $\chi_{e 1}\left(x^{\prime}, z^{\prime}\right)=$ $\chi_{e}\left(x^{\prime}, z^{\prime}\right) / \sqrt{z^{\prime}}$ with respect to $x^{\prime}$ and $z^{\prime}$, and $\widehat{\hat{\chi}}_{m 1}(\eta(p), \varsigma(p))$ is the double Fourier transform of the auxiliary function $\chi_{m 11}\left(x^{\prime}, z^{\prime}\right)=\chi_{m 1}\left(x^{\prime}, z^{\prime}\right) / \sqrt{z^{\prime}}$. Both these transforms are calculated in the spectral point

$$
\begin{gathered}
\eta=p, \\
\varsigma=\sqrt{4 k_{s}^{2}-p^{2}} .
\end{gathered}
$$

The spectral weight and $f(p)$ is given by

$$
\begin{aligned}
f(p)=\frac{j k_{s}^{2} \sqrt{\pi} f I_{o} \mu_{o}^{2} \mu_{s} \exp (j(\pi / 4))}{\left(\mu_{o} k_{z s}(p / 2)+\mu_{s} k_{z o}(p / 2)\right)^{2}} \sqrt{\frac{k_{z s}(p / 2)^{3}}{(p / 2)^{2}+\left(k_{z s}(p / 2)\right)^{2}}} \\
\quad \times \exp (-j \Delta(p / 2)) \exp \left(j 2 k_{z o}(p / 2) z_{s}\right),
\end{aligned}
$$

where $f$ is the working frequency and $k_{z o, s}=\sqrt{k_{o, s}^{2}-p^{2}}$. 
The physical conclusion related to (9) is that a linear reconstruction will be substantially the same for dielectric and magnetic targets (we have a formal difference of the two object functions, but it is irrelevant), and in particular, any linear reconstruction will not able to distinguish whether the focused target was dielectric or magnetic (or both) contrasted with the surrounding soil.

\section{Experimental Tests}

The experimental test has been performed in a box set up within the Laboratory of Applied Geophysics of the Department of Biological and Environmental Sciences and Technologies of the University of Salento. The box is a wooden container internally sized $1.5 \times 1.18$ square meters and high $90 \mathrm{~cm}$ from the floor of the tank. It has been filled up with sand up to the top.

Within this box, four targets have been buried. Two equal metallic sheets sized $20 \times 25$ square $\mathrm{cm}$, and two equal polystyrene boxes, the first of which filled up with (dusted) magnetite and the second filled up with the same sand that fills the tank. The size of the box filled up with magnetite is $23 \times 16 \mathrm{~cm}^{2}$, whereas that of the box filled with sand is $26 \times 18 \mathrm{~cm}^{2}$. Both boxes are high $8.5 \mathrm{~cm}$ (actually their shape is not that of a perfect parallelepiped, because they are slightly larger at the central point of their height). The thickness of the polystyrene walls of the boxes is of the order of $4 \mathrm{~mm}$. The box filled with sand is a reference about the negligibility of the polystyrene walls of the boxes. In particular, if the walls of the box are really negligible, this box should be almost transparent and we can be confident that the signal scattered by the box filled up with magnetite is not substantially ascribable to the content of the box and not to the container. The two metallic boxes also were markers, and in particular they have been placed in two different points at two different depths, so to have a check also about the degree of homogeneity of the sand. Figure 2 illustrates the plant, the position of the targets, and the grid of the B-scan lines. In particular, the transect between two adjacent B-scan lines was $10 \mathrm{~cm}$.

The data have been gathered with a SIR-3000 system, manufactured by GSSI, by making use of an antenna with nominal peak frequency at $900 \mathrm{MHz}$, manufactured by IDS.

With regard to the characteristics of the magnetite, exploiting the model assessed in [14], we have estimated in the band of interest an average relative dielectric permittivity nearly equal to 11.8 and an average relative magnetic permeability about nearly to 2 -j0.5. By exploiting the metallic sheets we have evaluated that the sand is quite homogeneous and its relative permittivity can be assumed equal to 4 . Moreover, we have assumed for the inversions a conductivity of the soil equal to $0.001 \mathrm{~S} / \mathrm{m}$.

We have performed both a standard processing based on the Kirchhoff migration (performed by means of the commercial software Reflexw 15) and a tomographic inversion of the data, based on the described inverse scattering model $[11,12]$. We show the results at three constant depth slices, at the depth of about 40,50 , and $80 \mathrm{~cm}$, that is, at the depth of the four targets (see Figures 3, 4, 5, 6, 7, and 8).

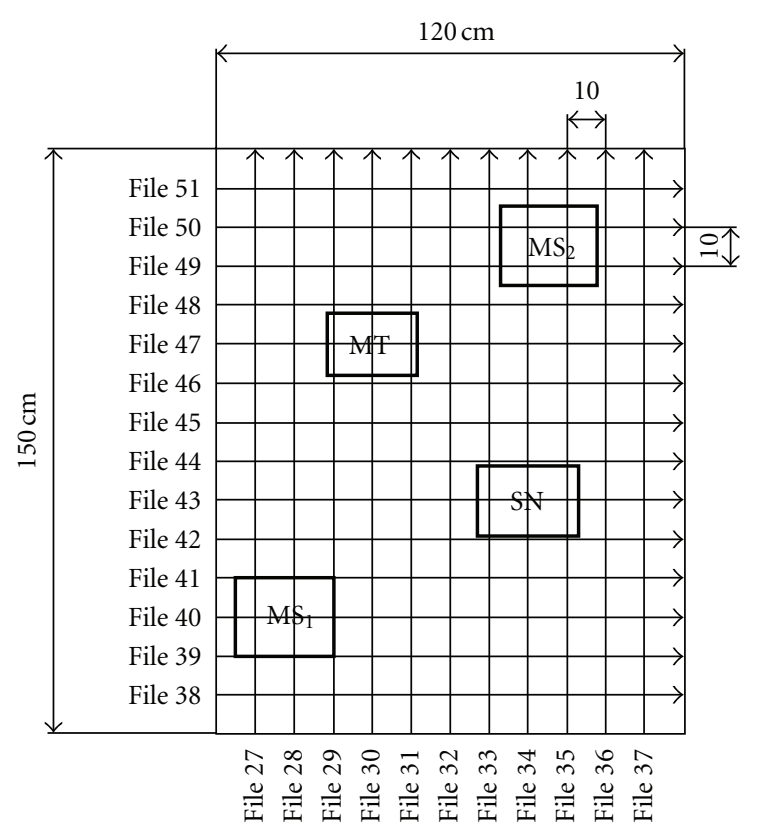

FIGURE 2: Plant of the box: the axes are in $\mathrm{cm}$, the first metallic sheet $\left(\mathrm{MS}_{1}\right)$ is at the depth of $50 \mathrm{~cm}$, the box filled up with magnetite (MT), and the box filled up with sand (SN) is at the depth of $41.5 \mathrm{~cm}$ (meant with respect to their top); the second metallic sheet $\left(\mathrm{MS}_{2}\right)$ is at the depth of $80 \mathrm{~cm}$.

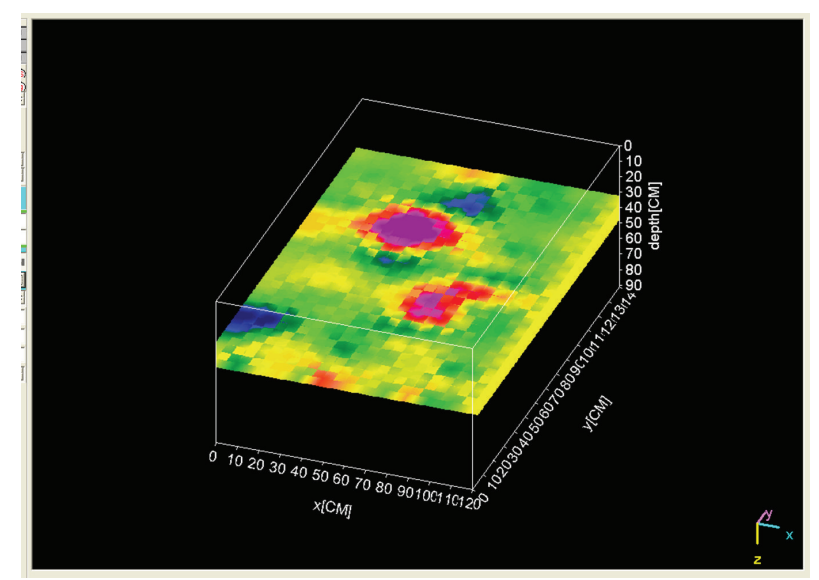

Figure 3: Constant depth at the depth of about $40 \mathrm{~cm}$, achieved from migration.

For the inverse scattering algorithm, we have made use of an investigation domain $110 \mathrm{~cm}$ long (two investigation domains have been joined side by side for the long edge of the tank, with some correspondent to zero padding of the data); the discretization of the investigation domain has been performed by means of 45 Fourier harmonic functions along the abscissa and 37 step functions along the depth; the working frequency band (based on the spectrum of the data) ranged from 550 to $1250 \mathrm{MHz}$, sampled with a frequency step of $50 \mathrm{MHz}$. The spatial step of the data was $1 \mathrm{~cm}$.

The comparison shows that the magnetic properties of one of the targets do not cause any particular effect in the 


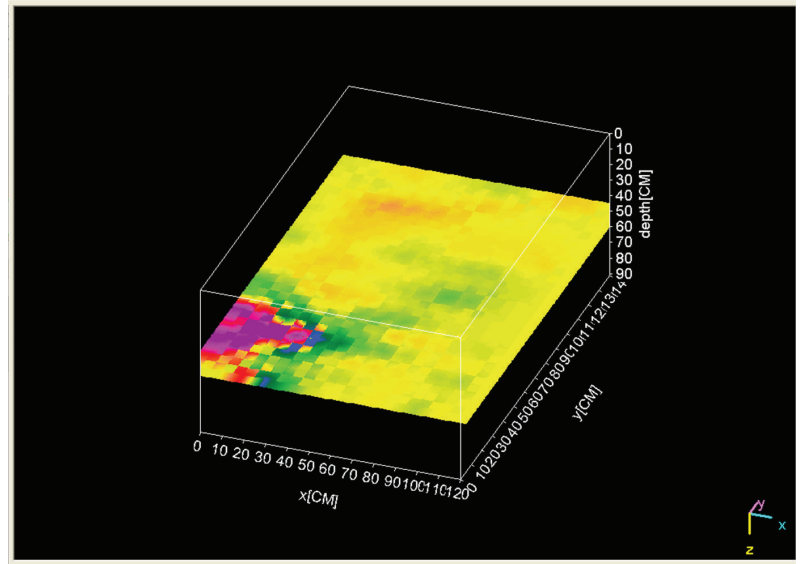

Figure 4: Constant depth slice at the depth of about $50 \mathrm{~cm}$, achieved from migration.

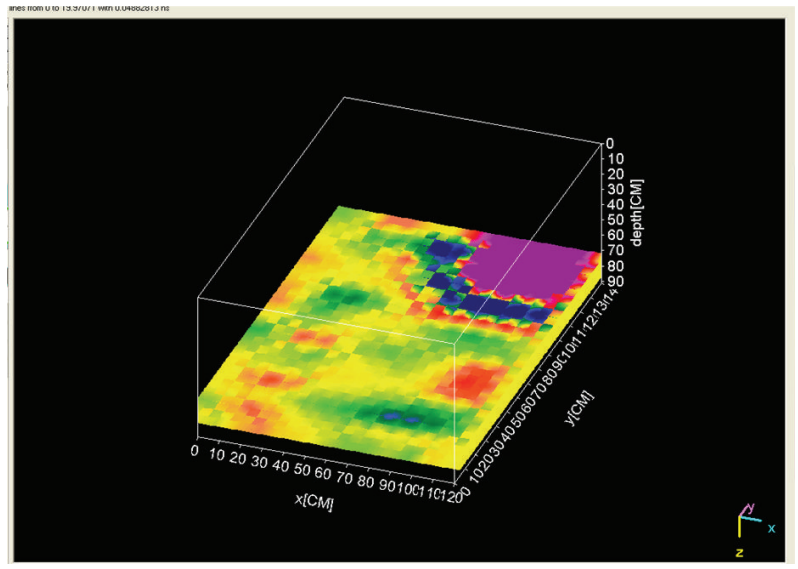

Figure 5: Constant depth slice at the depth of about $80 \mathrm{~cm}$, achieved from migration.

reconstruction, either in the migrated or in the inverted data. In particular, we can see more evident "spots" regarding the two boxes within the migrated data (Figure 3) than in the inverted data (Figure 6). However, the size of the two boxes is better reproduced in the inverted data, which also show more clearly the difference of scattered energy between the box filled with sand and that filled with magnetite. In both cases we have artefact, due to the metallic sheets and the edge effects of the tank. Moreover, the inversion is affected by the fact that the box has been (for computational reasons) in two adjacent investigation domains, which causes the horizontal seam visible in the middle of Figures 6-8. Also the shallower metallic sheets seem slightly better localised in the inverted data. However, the comparison suggests that, when possible, both processing schemes are worth performing, since each of them has its intrinsical pros and cons. The metallic sheets are focused in a poorer way than the boxes: this is because both of them are close to the edges of the tank (so that a limited view angle problem arises for them). In particular, with regard to the deeper metallic sheet the problem of the limited view angle added to that of loss of resolution with the depth makes

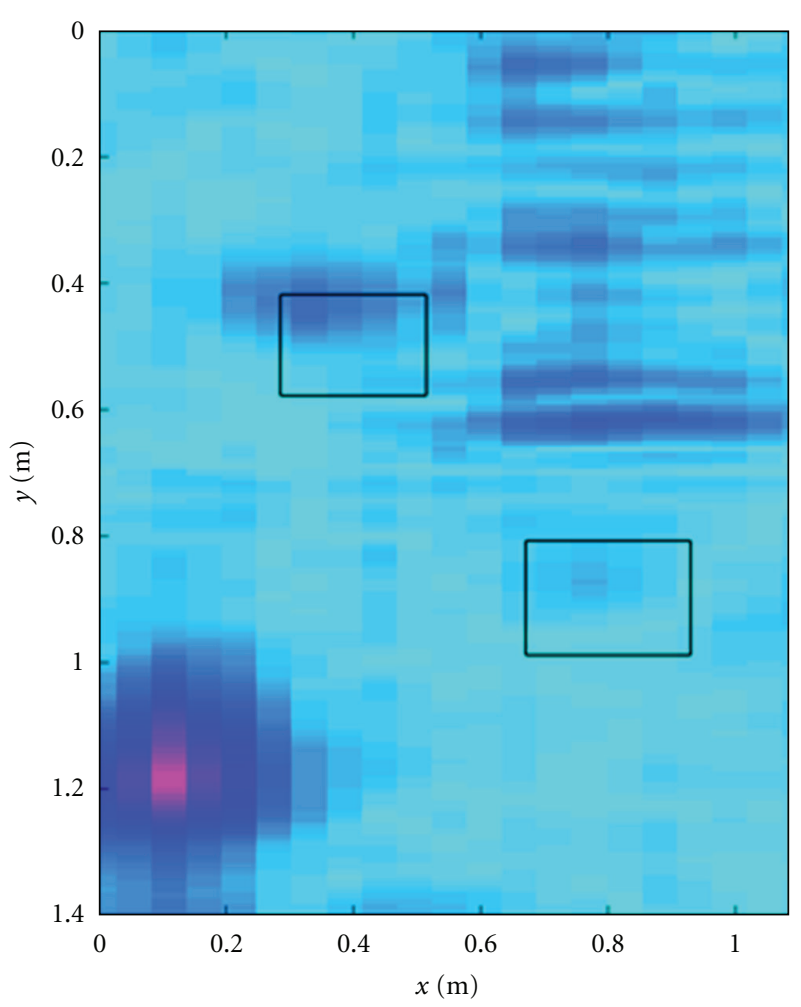

FIGURE 6: Constant depth slice at the depth of about $40 \mathrm{~cm}$, achieved from inversion.

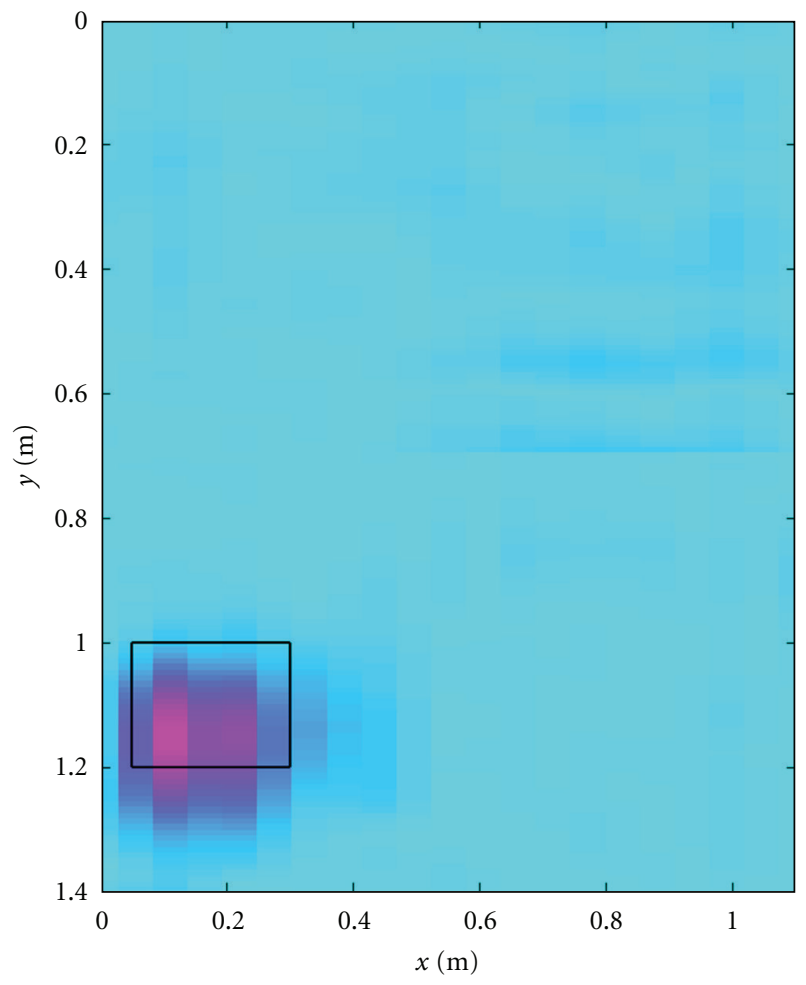

FIGURE 7: Constant depth slice at the depth of about $50 \mathrm{~cm}$, achieved from inversion. 


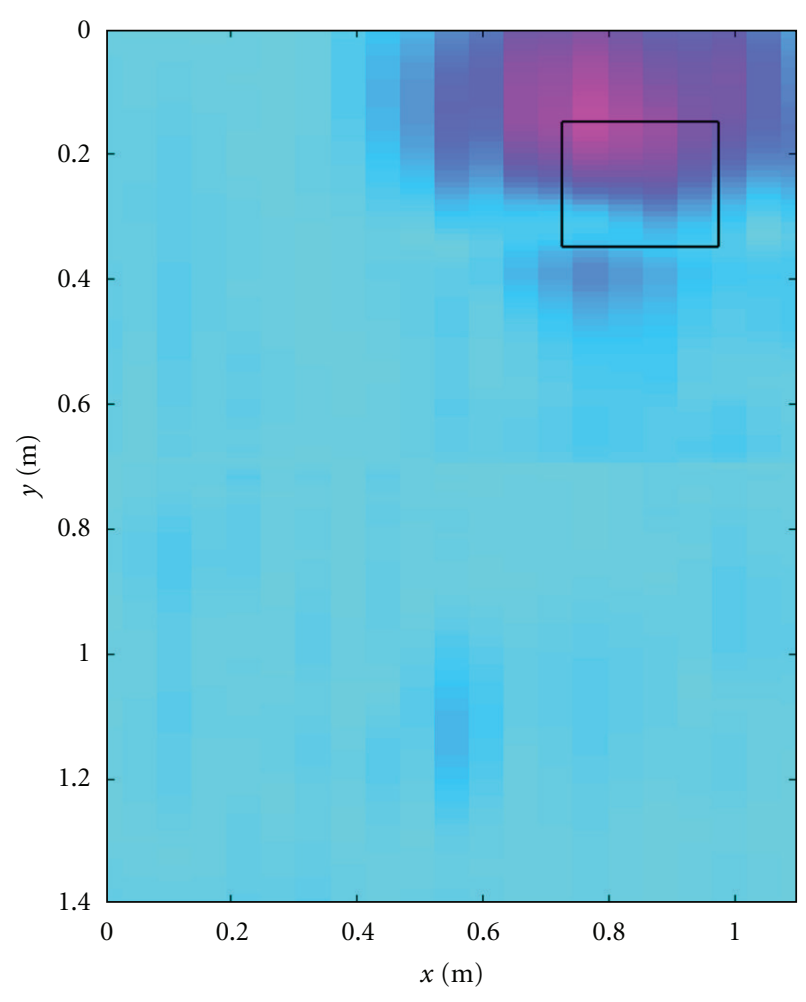

Figure 8: Constant depth slice at the depth of about $80 \mathrm{~cm}$, achieved from inversion.

the achieved focussing poorer than the one achieved for the shallower targets, both migrating or inverting the data (see Figures 5 and 8).

\section{Conclusions}

In this paper we have dealt with the scattering from buried targets that can exhibit both a dielectric and a magnetic contrast with respect to the surrounding soil. We have reported a resume of theoretical considerations more deeply exposed elsewhere [11], or even inedited (but the space required to expose them in a full way would have been too long). We have shown some pseudo 3D reconstructions, achieved from experimental data. In future applications, therefore, we will try to measure magnetic properties of the soil or of the targets by other methods, exploiting, for example, TDR and GPR data and/or nonlinear processing; this activity could be of interest in Mars and other planets' exploration $[8,9]$.

\section{Acknowledgment}

The authors thank Dr. Antonia Tamborino for her collaboration.

\section{References}

[1] D. Colton and R. Kress, Inverse Acoustic and Electromagnetic Scattering Theory, Springer, 1992.
[2] W. C. Chew, Waves and Fields in Inhomogeneous Media, IEEE Press, Piscataway, NJ, USA, 1995.

[3] P. Chen, R. X. Wu, T. Zhao, F. Yang, and J. Q. Xiao, “Complex permittivity and permeability of metallic magnetic granular composites at microwave frequencies," Journal of Physics D, vol. 38, no. 14, pp. 2302-2305, 2005.

[4] J. L. Young and C. M. Johnson, "A compact recursive transimpedance Green's function for the inhomogeneous ferrite microwave circulator," IEEE Transactions on Microwave Theory and Techniques, vol. 52, no. 7, pp. 1751-1759, 2004.

[5] G. Dural and M. I. Aksun, "Closed-form Green's functions for general sources and stratified media," IEEE Transactions on Microwave Theory and Techniques, vol. 43, no. 7, pp. 15451552, 1995.

[6] J. B. Jarvis, M. D. Janezic, B. F. Riddle et al., Measuring the Permittivity and Permeability of Lossy Materials: Solids, Liquids, Metals, Buildings Materials, and Negative-Index Materials, NIST Technical Note 1536, 2004.

[7] M. Nabighian, Electromagnetic Methods in Applied GeophysicsTheory, vol. 1, Society of Exploration Geophysics, 1987.

[8] D. E. Stillman and G. R. Olhoeft, "Electromagnetic properties of martian analog minerals at radar frequencies and martian temperatures," in Proceedings of the 37th Lunar and Planetary Science Conference, 2006.

[9] G. Picardi, J. J. Plaut, D. Biccari et al., "Radar soundings of the subsurface of Mars," Science, vol. 310, no. 5756, pp. 19251928, 2005.

[10] R. Persico and F. Soldovieri, "Two dimensional inverse scattering from buried magnetic anomalies," Annals of Geophysics, vol. 51, no. 1, pp. 147-158, 2008.

[11] R. Persico and F. Soldovieri, "Two-dimensional linear inverse scattering for dielectric and magnetic anomalies," Near Surface Geophysics, vol. 9, no. 3, pp. 287-295, 2011.

[12] R. Persico, F. Soldovieri, S. Negri, and E. Pettinelli, "Dielectric and magnetic anomaly imaging from GPR data," in Proceedings of the 13th Internarional Conference on Ground Penetrating Radar (GPR '10), Lecce, Italy, June 2010.

[13] W. Tabbara, B. Duchene, C. Pichot, D. Lesselier, L. Chommeloux, and N. Joachimowicz, "Diffraction tomography: contribution to the analysis of some applications in microwaves and ultrasonics," Inverse Problems, vol. 4, no. 2, pp. 305-331, 1988.

[14] K. Klein and J. C. Santamarina, "Ferromagnetic inclusions in geomaterials: implications," Journal of Geotechnical and Geoenvironmental Engineering, vol. 126, no. 2, pp. 167-179, 2000 . 

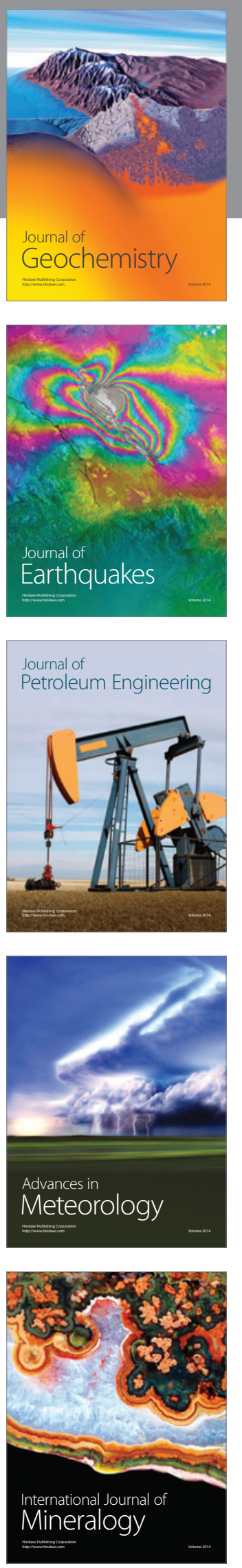
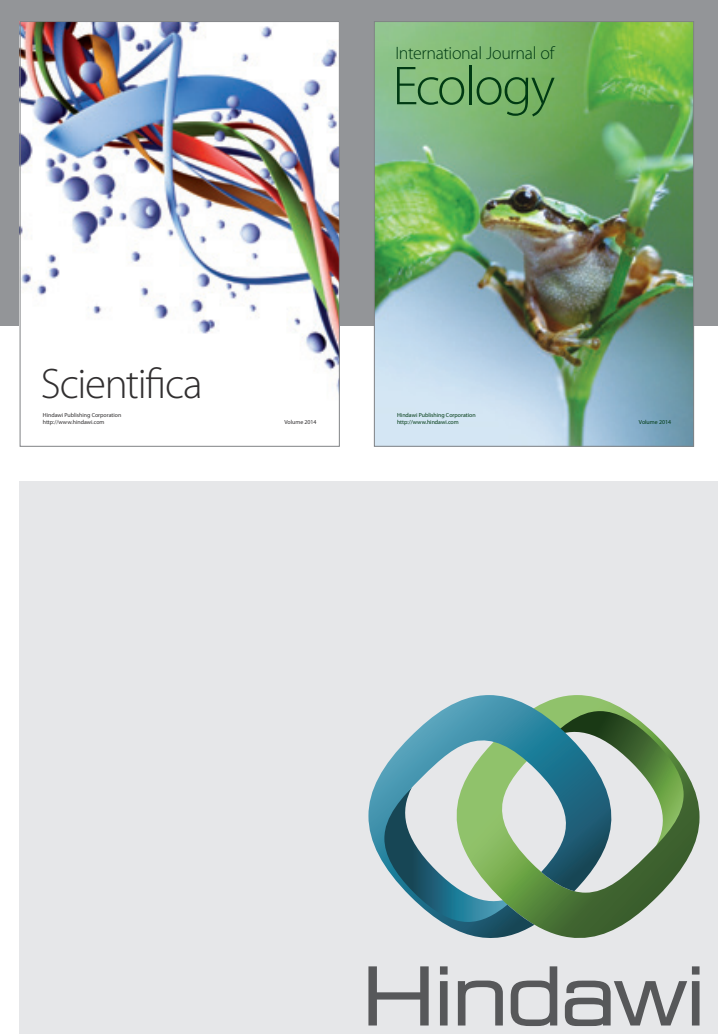

Submit your manuscripts at http://www.hindawi.com
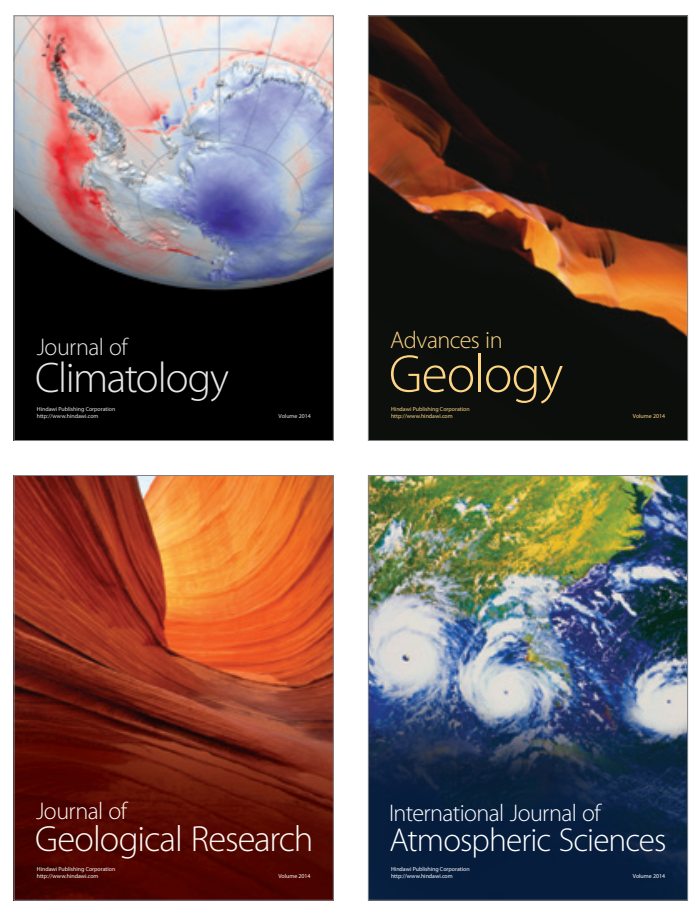
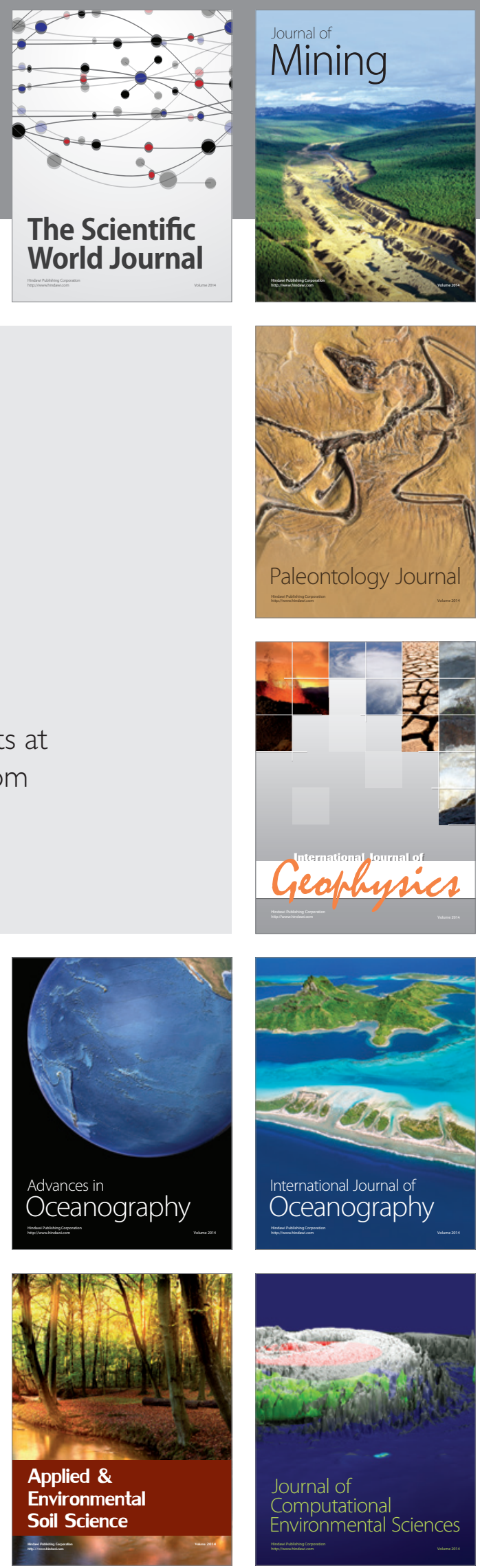